\title{
The Co-Evolution of Number Concepts and Counting Words $^{1}$
}

\author{
Heike Wiese, Humboldt-Universität Berlin \& Yale University
}

\begin{abstract}
Humans possess a number concept that differs from its predecessors in animal cognition in two crucial respects: (1) it is based on a numerical sequence whose elements are not confined to quantitative contexts, but can indicate cardinal/quantitative as well as ordinal and even nominal properties of empirical objects (e.g. 'five buses': cardinal; 'the fifth bus': ordinal; 'the \#5 bus': nominal), and (2) it can involve recursion and, via recursion, discrete infinity. In contrast to that, the predecessors of numerical cognition that we find in animals and human infants rely on finite and iconic representations that are limited to cardinality and do not support a unified concept of number.

In this paper, I argue that the way such a unified number concept could evolve in humans is via verbal sequences that are employed as numerical tools, that is, sequences of words whose elements are associated with empirical objects in number assignments. In particular, I show that a certain kind of number words, namely the counting sequences of natural languages, can be characterised as a central instance of verbal numerical tools.

I describe a possible scenario for the emergence of such verbal numerical tools in human history that starts from iconic roots and that suggests that in a process of co-evolution, the gradual emergence of counting sequences and the development of an increasingly comprehensive number concept supported each other.

On this account, it is language that opened the way for numerical cognition, suggesting that it is no accident that the same species that possesses the language faculty as a unique trait, should also be the one that developed a systematic concept of number.
\end{abstract}

\section{Introduction}

What role does language play in numerical thinking? This paper approaches this question from an evolutionary perspective and 
investigates the impact of our language faculty on the emergence of a fully-blown, unified number concept in human development. I describe a route to number that suggests that numerical thinking developed in a pattern of co-evolution of number concepts and counting words, indicating that language played a pivotal role in the emergence of systematic numerical cognition in humans.

This article has four main parts. Section 1 provides the background for our discussion and describes the distinctive characteristics of the human number concept. On this basis, section 2 looks into the evolutionary foundations of number: it shows what cognitive resources the development could build on. I discuss, on the one hand, evolutionarily old concepts that we share with other species, and on the other hand, genuinely human cognitive capacities that are supported by our language faculty. Section 3, the main section of this article, shows how a systematic number concept might have emerged in human history, based on these foundations. I present a developmental account that suggests that the gradual emergence of a systematic number concept was supported by the similarly gradual development of a more and more sophisticated counting sequence. In section 4, I summarise the main points of my account and discuss its implications for a view of numerical cognition and numeral semantics and the language-number relationship. 


\section{The human number concept}

As a background for our discussion of number evolution, this section makes clear what is involved in human numerical cognition, that is, what is meant when we talk about a 'systematic number concept' in humans. I am going to address three questions: what do we use numbers for, how do we use them, and what kind of entities do we use as numbers?

\subsection{What we use numbers for}

When one thinks of numbers, the first thing that comes to mind is usually cardinality: numbers can be used to indicate 'how many', and it is this cardinal aspect of number usage that most accounts of numerical thinking and numeral semantics focus on. However, cardinality is not the only property we can indicate with numbers. What makes numbers so special, and so powerful as mental tools, is that we can use them to indicate a broad range of empirical properties, of which cardinality is just one - certainly a salient one, but definitely not the only property we assess in number assignments. We can distinguish three main kinds of number assignments:

(i) In cardinal number assignments, numbers indicate the cardinality of sets, they identify a numerical quantity as in 'five buses', where the number indicates the cardinality of a set of buses. Building on this kind of usage, numbers can also be employed to 
indicate abstract cardinalities in arithmetic contexts (' $3+2=5$ '), and to indicate, together with measure units, properties like length, weight, or temperature ('5 cm long', '5 kg of apples', ' 5 C C'). Since both arithmetic and measurement contexts can be derived from cardinal assignments where the number indicates a numerical quantity, ${ }^{2}$ I will concentrate on these as the central case in point.

(ii) In ordinal number assignments, numbers indicate the position of an element in a sequence, they identify a numerical rank as in 'the fifth bus'. In this example, the number identifies the position of a particular bus in a sequence of buses, for instance a position in the line of buses arriving at a depot.

(iii) In nominal number assignments, numbers indicate the identity of an element within a set, as in 'the \#5 bus'. Here, the number works as a label to identify a particular bus (or bus line) within a set of buses.

\subsection{How we use numbers}

The reason why numbers have such a wide range of applications, the secret of their success, so to speak, is a pattern of association that I will call system-dependent linking, or for short dependent linking: in number assignments, the link between a number and an object does not draw on features they have as individuals, but instead it depends on the positions they have in their respective systems. Numbers and objects are not treated as individuals, but 
stand for corresponding places or properties in a numerical and an empirical relational structure. ${ }^{3}$ This means that first and foremost, we associate relations in number assignments. Let me make clear what that means by identifying the different relations we associate in each kind of number assignment.

In cardinal number assignments, we associate the numerical relation '>' with the empirical relation 'has more elements than': if one set of buses gets the number 5 ('five buses'), and another set gets the number 6 ('six buses'), we know that the second set has more elements, because $6>5$.

In ordinal number assignments, we associate the numerical relation '<' with sequential empirical relations like 'arrives before', 'is faster than', etc.: if one bus receives the number 5 ('the fifth bus'), and another one receives the number 6 ('the sixth bus'), we can deduce that the first one arrived earlier at the depot, because $5<6$. In nominal number assignments, we associate the numerical relation ' $=$ ' (or ' $\neq$ ', respectively) with the empirical relation of (non-)identity: if one bus receives the number 5 ('the \#5 bus'), and another one receives the number 6 ('the \#6 bus'), we expect them to go on different lines, because $5 \neq 6$.

Given these different ways in which we employ them, what can we then identify as the essential properties of numbers? When we have a look at the relations that we draw on in each case, it turns out that we do not have to ask much of our numbers: in order to work in 
nominal number assignments, they have to be well-distinguished, so that the relation ' $=$ ' (or ' $f$ ') holds; for cardinal and ordinal number assignments, they have be ordered within a progression, so that the relation ' $>$ ' or ' $<$ ' identifies sequential positions. If we want to make sure that there are no limits to the size of the empirical structures that numbers can be assigned to, we might want to add as a third requirement that our number sequence should be infinite.

This supports a criteria-based approach to numbers, an approach that sees numbers as tools that are used to assess different (cardinal, ordinal, and nominal) properties in empirical objects, and as such tools, must satisfy certain criteria: a number sequence, we can say, is a sequence that fulfills three requirements ${ }^{4}$ - it must (1) have well-distinguished elements, (2) be a progression, (3) be infinite and this sequence must be applied to empirical objects in a pattern of dependent linking.

Adopting such a criteria-based approach means, then, that when we identify a sequence that fulfills our number criteria, this sequence can be used as a number sequence; its elements can be employed as numerical tools. Hence, 'tools' here is used in the same way we use it in everyday speech, say, when talking about tools like hammers. For an object to be successfully used as a hammer, it has to satisfy certain criteria (e.g. be of a sufficiently hard material, have a handle etc.); if it satisfies these criteria and if we actually use it as a 
hammer, say, in order to drive a nail into a wall, then this object is a hammer in the sense that it is used as a tool for hammering. Similarly, if a sequence satisfies the number criteria we identified above and if it is used to assess empirical properties in a pattern of dependent linking, then this sequence is a number sequence in the sense that it is used as a tool in number assignments.

Hence, we are not looking for abstract entities called 'numbers', which, following the traditional approach, would be defined as sets of sets (or as sets of collections, cf. Hurford 1987). Defining numbers as sets or collections specifies them as cardinal, something I believe should be avoided, given their status as flexible tools that cover cardinal, ordinal, and nominal contexts. What we are looking for is a progression that fulfills our criteria for numerical tools, and this means, among other things, that it is not specialised for applications to sets, but can be used in non-cardinal contexts as well. What sequences can we now identify as such numerical tools?

\subsection{What numbers we use}

A good candidate for the job of numbers is a certain kind of number words, namely the counting sequences of natural languages: sequences of words like 'one, two, three, four, ...'. As words, they are well-distinguished from each other by their phonological representations, fulfilling the first requirement for numbers we identified above. Unlike other words, though, they also 
meet the other two conditions on numbers: they are ordered within a progression, and this progression is infinite, that is, for any given element of a counting sequence, one can always tell its successor. In order to achieve this constructive infiniteness, a counting sequence makes use of linguistic recursion: applying recursive lexical rules, one can derive numerals from numerals from numerals ..., which ensures that the sequence will never run out of elements. $^{5}$

Their sequential order distinguishes counting words from other, non-numerical words and is responsible for their exceptional status within the linguistic system. ${ }^{6}$ But while it makes counting words outsiders in language, this feature is at the core of the numerical side of their personality: it is the crucial characteristic we draw on when we use them as numbers.

And it is counting words we actually employ in every-day number assignments: we do not use them to denote something, but rather, to do something, namely to figure out cardinalities, sequential ranks, and identities in the course of number assignments. For instance, in order to find out how many buses are in a parking lot, one would match up each bus with a counting word, starting with 'one' and using them in their sequential order, such that the first bus that one counts receives the word 'one', the second receives the 'two', and so on, until the last receives, say, the word 'twelve'. This number word is then used to indicate the cardinality of the 
whole set, and it can do so because of its sequential position in the counting sequence.

This differs radically from what we usually do with words. In particular, counting words are not used for naming objects, they do not fulfill referential purposes, as other words do: one does not mean to name the first bus 'one', the next one 'two' etc. Rather, one uses counting words as gadgets to figure something out.

Taken together, this means that counting words are not names for numbers, but verbal numerical tools: they form a progression of well-distinguished entities with an instrumental, non-referential status in number assignments. As a result, counting words have a dual status: on the one hand, they are verbal elements, on the other hand, they are numerical tools.

The special status of counting words as entities correlating the linguistic and the numerical domain has been emphasised in other accounts of numerals and numerical thinking as well, most notably by Hurford (1987), who points out that "numeral systems lie in the intersection of the human language faculty and the number faculty” (p.3). Note, though, that according to the account I developed here, the dual status of counting words crucially means that they are numbers (as well as words), rather than number names, that is, they do not refer to extra-linguistic 'numbers', but instead are used as numbers right away. 
In contrast to that, Hurford (1987) follows the traditional approach by assuming two separate systems: a system of numbers (defined as sets of collections), and a system of numerals that denote them; he characterises numeral systems as "systems of names" (p.12), and numbers as "the non-linguistic entities denoted by numerals" (p.178). The way in which language contributes to numeracy under such an account is less fundamental than what I suggest here: if numerals are names for numbers, language can help us develop such names and hence allows us to reason about numbers, ${ }^{7}$ but it does not give us numbers directly, within the linguistic system. ${ }^{8}$

If, on the other hand, we identify counting words as verbal numerical tools, this then means that language gives us instances of numbers, words that we can employ as numbers, rather than just as names that we employ to denote numbers and to reason about them. But how can such tools emerge if we do not have a number concept already, a concept that allows us to grasp the logic of number assignments? In the following two sections, I describe the foundations that the emergence of numerical tools could build on, and then I develop a possible scenario for the gradual co-evolution of counting sequences and numerical concepts. 


\section{Foundations for the emergence of numbers}

What do we need for our number concept to emerge? More specifically, what was already there before numbers emerged, and what had to develop still?

Based on the discussion in the previous section, we can identify two cognitive systems that are relevant for our number concept. System 1 supports the grasp of empirical properties such as the cardinality of sets, the position of elements in a sequence, and the identity of elements in a set. System 2 supports the grasp of numbers as tools that are used in a pattern of dependent linking to assess these properties in empirical objects. It is this second system that makes the human number concept so powerful; it brings together cardinal, ordinal, and nominal relationships between empirical objects under the umbrella of number assignments, and allows us to assess cardinalities (as well as ranks and identities) well beyond the limits of our perception.

The development of these two systems can draw on cognitive capacities coming from different sources: system 1 is supported by early concepts of empirical properties that we share with other species; system 2 is uniquely human and makes use of a cognitive pattern provided by the human language faculty. 


\subsection{Prenumerical concepts of quantity, rank, and identity}

In order to grasp the empirical properties we assess in number assignments, we can make use of evolutionarily old capacities that have been found in other species, too. Mammals and birds have been shown to distinguish small sets of different sizes, and to perform simple transformations on them, indicating a prenumerical, biologically determined concept of cardinality. ${ }^{9}$ Another gift from our mental heritage is the apprehension of serial order, and related to this - of an object's position within a progression. Again, this capacity was demonstrated in a number of experiments with different animal species, among them canaries, rats, monkeys, and apes. $^{10}$

Similar evidence comes from human infants. In particular, newborns have been shown to discriminate small set sizes, ${ }^{11}$ and babies as young as 5 months are able to perform transformations on small sets. ${ }^{12}$ Infants have also been shown to possess a (possibly domain-specific) knowledge of physical objects that enables them, among others, to pick out discrete objects in their environment and to represent some of their properties even when the objects are moved out of their view, ${ }^{13}$ indicating an early basis for the distinction (identity versus non-identity) of objects.

Note, though, that these are prenumerical concepts of empirical properties, rather than numerical capacities: they provide the cognitive underpinnings to grasp the relevant empirical properties 
in number assignments - set size (cardinal), sequential rank (ordinal), and identity (nominal) - but they do not support numbers themselves, the tools we use to assess these properties in a pattern of dependent linking between two relational structures. In particular, prenumerical representations of set sizes are supported by iconic mental representations: they draw on 'object files', mental tokens that are filed in short-term memory and work like tallies, representing the elements of a set. ${ }^{14}$ This way, object files allow us to discriminate between sets of one, two, and three elements and to perform simple transformations on them by way of iconic representations: a set of object files represents elements of an empirical set, but it is not part of a numerical relational structure, and the representation does not involve any dependent linking.

\subsection{Non-numerical dependent linking}

The cognitive faculty that endows us with the capacity for dependent linking is a uniquely human domain: our language faculty. Language is characterised by a combination of arbitrary links between individual signs and their referents, and non-arbitrary (namely, diagrammatically iconic ${ }^{15}$ ) links between the structure of complex signs and their meaning that allow us to produce and understand an unlimited number of complex constructions. 
This is possible because the interpretation of complex linguistic signs is based on an association of relations: we associate relations between signs with relations between their referents. For instance, in order to find out that in a sentence like 'The dog bites the rat.' the dog is the biter and the rat is the one who gets bitten, we do not need to learn these correlations by heart. Rather, we associate signsign relations like subject of, and object of with conceptual relations like agent of and patient of.

Hence, in language, we do not focus on individual signs and individual referents, but on their positions within their respective systems. The link between 'dog' and agent and 'rat' and patient does not draw on features of 'dog' as an individual word, but is dependent on its position within the sentence: a linguistic instance of dependent linking. This association of relations is a central feature of language as a human faculty, the feature that allows humans to make the step from indexical signs to symbolic thinking. ${ }^{16}$

Ultimately, this means that language provides a cognitive pattern that can be used in the application of numbers: in linguistic reference, a relational structure of signs is associated with a relational structure of (conceptualisations of) objects, just as in number assignments, we need a relational structure of numbers to be associated with a relational structure of objects. ${ }^{17}$ 
How do we get from one domain to the other, from a linguistic pattern of dependent linking to a numerical one? This is where counting sequences come in: as verbal sequences, they bring with them not only linguistic recursion - and hence, constructive infiniteness - but also the expectation to be assigned to objects in a pattern of dependent linking. Due to their dual status, counting sequences make an ideal vehicle to take us from one cognitive domain, language, to the other, number. ${ }^{18}$ Once we use a sequence of words for instrumental, rather than referential purposes, it is only a small step towards a full-blown number concept.

So, how could such sequences emerge? The following section describes a possible scenario for the evolution of verbal numerical tools, showing how the gradual emergence of counting sequences and the development of a more and more comprehensive number concept could have supported each other.

\section{An evolutionary scenario}

We can think of the co-evolution of number concepts and counting sequences as a development in four main stages. Stage 1 starts with iconic representations of cardinality. These representations can be non-verbal (like notches), or verbal, that is, constituted by words. At stage 2, the elements of some verbal iconic representations (that is, words) appear in a stable order, supported by their correlation with body parts, in particular with fingers, that are also used for 
cardinal icons. At stage 3, this stable order supports indexical links between individual words and individual cardinalities. At stage 4, these indexical links give rise to dependent links: a counting sequence is born. The pattern of association can now be generalised to cover non-cardinal as well as cardinal contexts, supporting a full-blown, unified number concept. The following sections describe these stages in more detail.

\subsection{Stage 1: Iconic roots}

Numerical development begins with a strictly prenumerical stage: no number assignment takes place yet. Instead, we find iconic representations of cardinality, that is, representations that are based on similarity. Such representations do not involve dependent linking, but are supported by the mental object files I mentioned above: mental tokens that are stored in short-term memory. We store one object file for each element of the represented set, hence the set of object files has the same cardinality as the set of objects it represents. By drawing on this similarity, the set of object files works as some kind of mental tallies, that is, as an iconic representation of a set size. As we have seen in 2.1 above, this grasp of set sizes is an evolutionarily old capacity that is evident in human infants as well as in other species.

Typical iconic cardinality representations produced by humans are visual tallies. These tallies have the advantage to allow the 
representation of larger set sizes. They can be used by themselves, e.g. in the form of notches on a stick, but they can also be accompanied by verbalisations, that is, by some kind of verbal tallies. Such a combination of verbal and non-verbal tallies is known from early Australian cultures, for instance from Aranda, as described by Strehlow (ca. 1945):

"Seven,” for instance, was normally expressed by putting down in the sand seven strokes side by side, with the words "njinta n, na, arbuñ n, nana, arbuna n, nana, etc. ... kalla n, nanantma” (= "here is one, here is another, here is another, etc. ... here is the last “). “Ten” was expressed by putting up both hands and spreading out all ten fingers and saying "lakintja" = "this many.” (Strehlow ca.1945:103)

Evidence for an early stage of tally usage in human history comes from a 30,000 year old fossilised wolf's bone that was found at a mammoth hunters' base in Dolní Věstonice. Interestingly, the notches on such prehistoric finds - including the ones on that wolf's bone - come often in multiples of 5, suggesting an early use of fingers as 'body tallies' for iconic representations of cardinality (Absolon 1957). This is supported by modern-day evidence pointing to an influence of finger tallying on counting sequences, in particular

- the abundance of base-10 (or 5 or 20)-structures in the counting sequences of the world; 
- frequent diachronic links of counting words to expressions for fingers;

- diachronic links of counting words to expressions referring to the act of (un-)bending fingers. ${ }^{19}$

\subsection{Stage 2: The emergence of stable order}

The use of fingers (and other body parts) as tallies can lead to the emergence of a stable conventional order and hence give rise to a second stage in number development: when fingers are used to represent elements of another set, they tend to be singled out successively, following the natural order of fingers on each hand. This means that our cardinality representations are generated in a fixed, stable order. In this order one could, for instance, start with the thumb on one hand, go all the way to the little finger, and then use the fingers of the second hand in the same way. As the differences in finger counting in modern cultures show, other orders are possible as well, of course; what is important here is that the salient order of fingers on each hand will support $a$ convention for singling out individual fingers successively in a fixed order.

Note that, given that cardinality representations are still iconic at this stage, this order does not fulfill any numerical purpose yet. For an icon, it does not matter which of its elements - that is, which individual tally - is produced first and which one last, since in the end, the whole set of tallies stands for a set of objects. For instance, 
if we want to represent a set of five sheep iconically with our fingers, it does not matter in which order we unbend (or bend) our fingers, as long as every single sheep is associated with exactly one finger, so that in the end, the whole fist stands for the whole set of sheep.

Hence, we do not need to presuppose any additional numerical concepts for this development. All we need for stable order to emerge is the systematic use of fingers in cardinality icons.

Given that body tallies are frequently accompanied by verbal tallies (namely the names for the body parts in question), a stable conventional order of fingers used in cardinality icons will lead to a stable conventional order of words. A stable list of words, a conventional verbal sequence, will then represent a set of objects iconically, such that each object is associated with exactly one word in our list.

Such non-verbal tallies accompanied by verbal ones can be found in body counting systems in Papua New Guinea, for instance in the Oksapmin language (cf. Saxe 1981). In order to represent a set size in these systems, one points to body parts in a fixed order (starting with the thumb on one side, going up on the arm and all the way to the head and down again via the other arm to the other hand) while saying aloud the name of each body part, identifying as many body parts as there are elements of the empirical set one wants to represent. 
Evidence for an iconic usage of word lists can also be found in individual development, in the acquisition of counting words by young children. When children learn to count, they first go through a stage where they use sequences of counting words to represent the cardinality of sets. At this stage, a child will not respond with one number word when asked to tell the cardinality of a set, for instance, when asked to tell how many teddy bears she has in her room, but instead, she will give the whole sequence of words leading up to the final number. For instance, if there were four teddy bears, she would say "One, two, three, four”, but would then not use the final word in her count, "four", to indicate the number of the whole set. ${ }^{20}$ Only later, around $31 / 2$ years of age, do children learn to produce non-iconic cardinality representations and use single words to represent whole sets, based on dependent links that associate quantitative relations between set sizes with sequential relations between counting words. ${ }^{21}$ What could this transition from iconic to numerical representations be like in ontogenesis?

\subsection{Stage 3: Indexical links}

At the stage I described in the previous section, we find lists of words used in a stable, conventional order to represent set sizes iconically. This can give rise to a third stage in number development, where individual words acquire indexical links with 
certain cardinalities. The basis for this is the prominent status of the final element in a verbal list.

The final word in a sequence is always more salient and more accessible than the other. This leads, for instance, to 'recency effects' shown in memory experiments: the last word in a list can be better recalled and memorised than the others (probably based on a buffer in short-term memory). ${ }^{22}$

This leads to a prominent status of the final word that is used in an iconic cardinality representation. Once the words are used in stable order, for a set of a given cardinality, the same word will always come last and hence be particularly salient for the representation of this cardinality. This, then, will support the emergence of indexical links between individual words and sets of a certain cardinality.

Evidence for the significance of such links comes from diachronic associations between counting words and sets of a particular size, which can still be found in a lot of modern counting sequences, e.g. in languages as diverse as Russian (where 'sorok', forty, goes back to Old Nordic 'sekr', the word for furs that were traded in bundles of forty), and Malinke (where 'wáa', one thousand, derives from the word for standardised baskets containing 1000 cola nuts).

\subsection{Stage 4: Dependent linking and generalisation}

Given that we have indexical links between individual words and sets of a certain cardinality, we now have verbal representations of 
cardinality. It is then only a small step from indexical links to the dependent links we want for a full-blown number concept: because we are in language, we can make use of the linguistic pattern of dependent linking. Based on this pattern, indexical links between words and cardinalities can evolve into dependent links, such that sequential relations in our word list are associated with quantitative relations between sets.

According to Deacon's (1997) account of language evolution, the transition of indices into a symbolic linguistic system (i.e., a system of signs that is based on what I called 'dependent linking') was supported by ritual contexts. Ritualised routines involve the repeated correlation of (vocal or non-vocal) tokens with objects, often accompanied by pointing gestures, which sets up a context of indexical relationships. According to Deacon (1997), the redundancy involved in these routines supports an attentional shift from concrete, indexical sign-object associations to abstract signsign relationships. This way, the repetitive context of rituals can support the grasp of symbolic systems.

In a similar vein, repetitive routines can support the emergence of relational structures in number development. The use of stable word lists for our cardinality representations constitutes ritualised repetitive and conventionalised - counting routines that emphasise sequential relationships between the words involved. By doing so, they support a shift from individual words to a relational structure. 
Ultimately, this supports the transition from indexical links between individual words and cardinalities to dependent links between a relational structure of counting words and a relational structure of sets. Elements of these two relational structures can then be associated by way of dependent linking.

We now have a sequence of well-distinguished elements with an instrumental status in cardinality assignments. This means that the elements of this sequence constitute verbal numerical tools: a counting sequence is born.

So far, these tools are confined to cardinal number assignments. However, since these assignments are not based on individual similarities between signs and objects, but rather on an association of relations (given that we employ dependent linking), the way is open to generalise the usage of our numerical tools and apply them to other, non-cardinal contexts. For this generalisation, counting words keep the instrumental status they have in cardinal assignments, and they also still undergo dependent linking. All that is new is that the dependent links we need for our number assignment will now focus on non-cardinal relations, namely ordinal and nominal ones.

A second field ready for expansion is the size of our counting sequence. Since our numerical tools are verbal ones, we can use the full power of linguistic recursivity, the recursivity that counting words bring with them as lexical items. This allows us to generate 
infinitely many new counting words, based on a finite set of primitive elements and rules for their combination. This way, linguistic recursivity provides constructive infiniteness for our number sequence.

At this stage, then, we have fully functioning numerical tools: we have an infinite verbal sequence that is employed, by way of dependent linking, in order to assess cardinal, ordinal, and nominal relations between empirical objects.

\section{Conclusion}

In the approach I presented here I made three main points: I argued for a non-Platonic view of numbers and a distinction of numbers and cardinality, for the gradual emergence of number concepts starting from iconic roots and going hand-in-hand with the gradual emergence of counting sequences, and for a strong impact of the language faculty on the development of numerical thinking. By way of conclusion, let me summarise the significance of these points for a view of numerical thinking and numeral semantics and of the language-number relationship.

4.1 Non-Platonic view of numbers; distinction of numbers and cardinality

I have characterised numbers as tools that are used in different kinds of number assignments to identify empirical properties like 
set sizes, ranks in a sequence, and (non-)identity within a set. Such an account involves both weaker and stronger assumptions than popular accounts of number that form the basis for most analyses of numeral semantics.

It makes weaker assumptions in the sense that it does not presuppose any abstract entities 'numbers' that are somehow out there in the world and need to be grasped by us when we acquire a concept of number or understand the meaning of cardinal numerals. Instead, numbers are regarded as cognitive tools that are developed by humans. Our criteria-based approach identified the crucial properties for these tools: what we need our numbers to be, is a progression (preferably infinite) of well-distinguished elements. As I have argued in this article, this means in particular that counting sequences of natural languages are suitable candidates for the job of numbers: as words, they are well-distinguished from each other, and in contrast to other kinds of words, they are sequentially ordered and form an infinite list.

Crucially, this means that these numerals do not refer to numbers, but are numbers: they are not the names of non-linguistic entities 'numbers', but linguistic instances of numbers. And since numbers are not regarded as abstract entities, this means that counting words are verbal instances of numerical tools, that is, verbal tools we use in number assignments. 
While this makes the approach I put forward leaner than standard accounts of numerical thinking, in another respect, it makes stronger demands, namely by distinguishing cardinality and numbers. As I have argued here, numbers are flexible and powerful tools that are used in a pattern of dependent linking to assess a broad range of properties in empirical objects. In contrast to this, cardinality is one of these properties - and by no means the only one: cardinality is an empirical property that can be assessed with numbers, in the same way that ranks in a sequence and the (non-)identity of elements within a set are empirical properties that can be assessed with numbers (namely, in ordinal and nominal number assignments, respectively). Numbers are the tools we use to identify these different properties in empirical objects, they are not one of the properties.

One of the implications of this distinction is that evidence for early concepts of cardinality in animals and human babies is not interpreted as evidence for numerical thinking. Rather, I have argued, it indicates prenumerical iconic concepts. In human development, these concepts support the grasp of one of the empirical properties we assess with numbers.

\subsection{Gradual emergence of a systematic number concept}

The main part of this article was dedicated to showing how these cognitive tools we call 'numbers' could have been developed in 
human history. I presented a possible route to numbers that suggests a co-evolution of iconic elements developing into counting words, and cardinality representations developing into number concepts.

The crucial point shown by this scenario is that, if we understand counting sequences as verbal numerical tools (as sketched in section 1), we can account for number development as a development by gradual transitions, based on small evolutionary steps: a development that starts from iconic representations that we share with other species and leads to a systematic and comprehensive number concept. Let me summarise these transitions from one stage to the next.

The first stage draws on evolutionarily old iconic roots: it is characterised by non-verbal and verbal tallies that are supported by mental object files - mental tallies that allow us to grasp different set sizes and that we share with other species. At the second stage, the use of body tallies, and in particular the use of fingers, leads to a stable order in our tallying, and since body tallies are often accompanied by names for the respective body parts, this stable order can then be passed on to the verbal domain. If verbal tallies are used frequently, the salience of the last word in a list will support indexical links between individual counting words and particular set sizes, leading to stage three in number development: from a parallel usage of verbal and non-verbal cardinal icons, we 
have proceeded to using individual words from a list as indices for individual cardinalities. At the fourth stage of number development, ritual contexts emphasise word-word relations in our list of indices and thus support the emergence of dependent linking. At this stage, we draw on sequential relations between our words to represent quantitative relations between sets. This usage of words allows us to expand our number concept in two directions: (1) based on recursive linguistic rules, we can increase our word set and thus handle set sizes far beyond the limits of our senses and up to infinity; (2) since we associate relations and are not dependent on iconic or indexical links anymore, we can generalise the usage of our numerals to non-cardinal contexts, yielding a unified concept of number that encompasses cardinal as well as ordinal and nominal applications.

4.3 Impact of the language faculty on the development of numerical thinking

The approach I put forward characterises the human language faculty as a crucial factor in the emergence of systematic numerical thinking in our species. According to this approach, language contributes in three main ways to number development:

(1) Material. Verbal elements, namely the counting sequences of natural languages, are an important instance of numerical tools. It is words - used first for iconic cardinality 
representations, and later as fully-blown numerical tools in number assignments- that trigger the emergence of number in human development.

(2) Application. Language as a symbolic system provides a cognitive pattern of dependent linking that can be adapted for the application of words as tools in cardinal, ordinal, and nominal number assignments.

(3) Generation. Linguistic recursivity yields words from words and thus allows us to generate an infinite verbal number sequence.

The first two points are crucial for the development of a number concept: with counting words, language gives us verbal numbers, and with dependent linking, it gives us a way to use them in order to assess empirical properties in systematic number assignments. According to this account, language is a prerequisite for numeracy not only in the sense that it allows us to reason about numbers and to grasp concepts of higher numbers, but, more fundamentally, in the sense that it provides numbers directly, within the linguistic system itself: language provides a progression of verbal entities that fulfill the role of numbers in a pattern of dependent linking. These two aspects, being a progression and being applied by way of dependent linking (in order to assess cardinal, ordinal, and nominal properties of empirical objects), are part and parcel of what constitutes numbers. In comparison to this, the third way in 
which language supports numerical development, namely by providing linguistic recursivity, is rather less important for our number concept. Recursion lays the grounds for constructive infiniteness, but this is not a necessary feature of a sequence we want to use in number assignments: as long as we only cope with finite empirical relational structures, a finite numerical sequence will do fine, and accordingly, not all cultures have an infinite, recursive number system (although, given that they have recursivity in language, once the need arises they can develop an infinite number system, or implement one from another culture). Language supports the implementation of recursivity in the numerical domain, but it does not necessarily trigger it. Once recursivity is employed, though, this opens the way for infiniteness in our number sequence, and eventually for a whole new range of mathematical objects that go far beyond the positive integers.

Hence, with counting words and dependent linking, language provides the crucial elements for numerical thinking, it gives us numbers as powerful and flexible mental tools; with recursion, it provides the means for an optional, yet substantial, upgrade of these tools.

Taken together, this means that when looking at the emergence of number, we are looking at a fundamentally shared enterprise, a deeply interwoven development of numerical and linguistic aspects. This co-evolution of number concepts and number words 
suggests that it is no accident that the same species that possesses the language faculty as a unique trait, should also be the one that developed a systematic concept of number.

\footnotetext{
${ }^{1}$ I would like to thank Charles Stewart, James Hurford, and an anonymous reviewer for comments on a previous version of this article.

${ }^{2}$ Cf. Wiese (2003a: ch.1).

3 More precisely, number assignments are constituted by homomorphic mappings between a numerical relational structure and an empirical relational structure (cf. Krantz et al. 1971, Narens 1985).

${ }^{4}$ Of these requirements, only the first two are strictly necessary. As mentioned above, the third one, infiniteness, is only relevant once we want to avoid any limits for the range in which our numbers can be applied; as long as we only deal with finite empirical relational structures, we do not need infiniteness in our number sequence.

${ }^{5}$ For a detailed discussion of the recursive rules governing the generation of numerals cf. Hurford (1975; 1987:ch.5).

${ }^{6}$ Cf. also Hurford (1987:6): "In one clear respect, numerals are unlike almost anything else in language. Numeral expressions are ordered, in the counting sequence." The sequential order, as well as their non-referential status (which will be discussed below) makes counting words also different from other words in first language acquisition, cf. Wiese (2003a: ch.3).

${ }^{7}$ Cf. for instance Hurford (1987:306): "The capacity to reason about particular numbers, above about 3 , comes to humans only with language”.

${ }^{8}$ Hence, while, like the present account, Hurford (1987) emphasises the importance of language for the development of numerical thinking, unlike the account I develop here, he characterises language as "the mental tool by which we exercise control over numbers." (p.305), rather than the system that provides numbers directly.

${ }^{9}$ Cf. Dehaene (1997), Carey (1998), Butterworth (1999) for overviews.

${ }^{10}$ Cf. Pastore (1961) for experimental evidence on canaries; Davis \& Bradford (1986) and Suzuki \& Kobayashi (2000) on rats; Orlov et al. (2000) on monkeys, and Kawai \& Matsuzawa (2000) on apes.

${ }^{11}$ Antell \& Keating (1983).

${ }^{12}$ Wynn (1992).

${ }^{13}$ Cf. Spelke (1990).

${ }^{14}$ For a discussion of object files cf. Mix (1999), Uller et al. (1999), Carey (2001). In order to represent the cardinality of a set, object files must be applied to collections of objects that are subsumed under a certain concept. For this kind of set formation, early salient concepts as physical object or discrete sound are
} 
already available in prelinguistic infants in the first months of life. As a study conducted by Wynn et al. (2002) showed, five-month-old infants might even be able to employ concepts as sophisticated as group in cardinality judgments.

In addition to object files, a second mental system can be employed in the grasp of quantities, namely that of analog magnitudes (cf. Whalen 1999; Gallistel \& Gelman 2000, Feigenson et al. 2004, Nieder 2005). Analog magnitudes are based on representations of accumulated quantity. Since in contrast to object files, such representations are fuzzy rather than discrete, I focus on object files for the evolution of number concepts that involve representations of discrete cardinality. Note that just like object files, analog magnitudes provide iconic, rather than numerical representations of set sizes (cf. Wiese 2003b for a detailed discussion). ${ }^{15}$ Cf. Peirce (1931: 2.277).

${ }^{16}$ Cf. Deacon (1997) for an account of symbolic thinking in human evolution.

${ }^{17}$ Note that this kind of linguistic support differs from linguistic bootstrapping of the kind suggested by Carey (2004): according to Carey (2004), in language acquisition, children integrate representations of natural language quantifiers with the counting sequence, to the effect that cardinal meanings of higher counting words can be constructed by successively adding 'one more' to the meaning of their successors in the count list (similarly to the developmental account proposed by Hurford 1987). In contrast to this, the present approach aims to capture the full flexibility of numbers, with cardinal, ordinal, and nominal usages (rather than meanings) alike. The central question here is therefore not so much how we arrive at cardinal interpretations for individual numerals, but rather, how a general pattern could emerge that allows us to use positions in the count list to assess different those kinds of empirical properties in number assignments. This is the pattern I identify as dependent linking.

${ }^{18}$ Note that this also solves a puzzle pointed out by Bloom (1994), namely how recursivity in language could have supported the emergence of recursivity in the numerical domain, given that recursivity is a holistic property of systems and cannot be transferred from one domain to the other. Following the approach developed here, recursivity could be passed on from language to number because it did not have to be transferred as an isolated feature, given that we use counting words as verbal numerical tools, that is, given that we employ words as numbers: in order to use, in the numerical domain, the recursivity that language provides, one does not have to move out of the system in the first place because one can use linguistic recursivity right away.

${ }^{19}$ Cf. e.g. Petitot (1876) and Menninger (1958) on Dëne Sų łiné, a language from the Northern Branch of the Athapaskan family spoken in North America. According to Menninger's account, the words for 'one' to 'five' go back to expressions meaning 'the end is bent' (little finger, one), 'it is bent once more' (ring finger, $t w o$ ), 'the middle is bent' (middle finger, three), 'only one remains' (thumb, four), and 'my hand is finished' (five). Butterworth (1999:61) describes a similar phenomenon for Zulu number words. 
${ }^{20}$ In an experiment conducted by Fuson \& Mierkiewicz (1980; reported in Fuson \& Hall 1983), some children, when asked "How many" again, repeated the word list as often as seven times, but would not give the last word as an answer.

${ }^{21}$ This is when children acquire what Gelman \& Gallistel (1978) called the 'Cardinality Principle'. Cf. Gelman (1990), Gallistel \& Gelman (1990), and Wynn (1990, 1992b, 1998) on individual numerical development and the different stages that children go through. The pattern of numerical development in phylogenesis is beyond the scope of this paper; in Wiese (2003a: ch.5), though, I give a detailed discussion of these stages and of the role of linguistic development in the transition from iconic cardinality representations to numerical representations based on dependent linking.

${ }^{22}$ On recency effects cf. Murdock (1962), Neath (1993), and Baddeley (1998). 


\section{References}

Absolon, K. 1957. "Dokumente und Beweise der Fähigkeiten des fossilen Menschen zu Zählen im mährischen Paläolithikum” ['Documents and proofs of fossil man's abilities to count in the Moravian paleolithicum']. Artibus Asiae (Institute of Fine Arts New York University) XX (2/3): 123-150.

Antell, S. E., and Keating, D. P. 1983. "Perception of numerical invariance in neonates”. Child Development 54: 695-701.

Baddeley, A. D. 1998. Human Memory: Theory and Practice, revised ed. Hove: Psychology Press.

Bloom, P. 1994. "Generativity within language and other cognitive domains”. Cognition 51: 177-189.

Butterworth, B. 1999. The Mathematical Brain. London: Macmillan.

Carey, S. 1998. "Knowledge of number: its evolution and ontogeny”. Science 282: 641-642.

Carey, S. 2001. "Cognitive foundations of arithmetic: evolution and ontogenesis”. Mind \& Language 16: 37-55.

Carey, S. 2004. "Bootstrapping \& the origin of concepts". Daedalus Winter 2004: 59-68.

Davis, H., and Bradford, S. A. 1986. "Counting behavior by rats in a simulated natural environment”. Ethology 73: 265-280. 
Deacon, T. W. 1997. The Symbolic Species. The Co-evolution of Language and the Brain. New York: Norton \& Co.

Dehaene, S. 1997. The Number Sense: How the Mind Creates Mathematics. Oxford: Oxford University Press.

Feigenson, L., Dehaene, S., and Spelke, E. S. 2004. “Core systems of number”. Trends in Cognitive Sciences 8 (7): 307-314.

Fuson, K. C., and Hall, J. W. 1983. "The acquisition of early number word meanings: a conceptual analysis and review”. In The Development of Mathematical Thinking, H. P. Ginsburg (ed), 49-107. New York: Academic Press.

Gallistel, C. R., and Gelman, R. 1990. "The what and how of counting. Cognition 34 (2): 197-199.

Gallistel, C. R., and Gelman, R. 2000. "Non-verbal numerical cognition: from reals to integers.” Trends in Cognitive Sciences 4(2): 59-65.

Gelman, R. 1990. "First principles organize attention to and learning about relevant data: Number and the animateinanimate distinction as examples”. Cognitive Science 14 (1): 79-106.

Gelman, R., and Gallistel, C. R. 1978. The Child's Understanding of Number. Cambridge, Mass.: Harvard University Press.

Hurford, J. R. 1975. The Linguistic Theory of Numerals. Cambridge University Press: Cambridge. 
Hurford, J. R. 1987. Language and Number: The Emergence of a Cognitive System. Blackwell: Oxford.

Kawai, N., and Matsuzawa, T. 2000. "Numerical memory span in a chimpanzee”. Nature 403 (6765): 39-40.

Krantz, D. H.; Luce, R. D.; Suppes, P., and Tversky, A. 1971. Foundations of Measurement. Academic Press: New York. Vol. 1.

Menninger, K. 1958. Number Words and Number Symbols: A Cultural History of Numbers, translated by Paul Broneer from the revised German edition (Cambridge, Mass.: MIT Press, 1969).

Mix, K. 1999. "Preschooler's recognition of numerical equivalence: sequential sets”. Journal of Experimental Child Psychology 74: 309-332.

Murdock, B. B. 1962. "The serial position effect in free recall”. Journal for Experimental Psychology 64: 462-488.

Narens, L. 1985. Abstract Measurement Theory. Cambridge, Mass.: MIT Press.

Neath, I. 1993. "Distinctiveness and serial position effects in recognition”. Memory and Cognition 21: 183-195.

Nieder, Andreas 2005. "Counting neurons: the neurobiology of numerical competence." Nature Reviews Neuroscience [Advance online publication 15 February 2005, doi:10.1038/nrn1626]. 
Orlov, T.; Yakovlev, V.; Hochstein, S., and Zohary, E. 2000. "Macaque monkeys categorize images by their ordinal number”. Nature 404 (6773): 77-80.

Pastore, N. 1961. "Number sense and 'counting' ability in the canary”. Tierpsychologie 181: 561-573.

Peirce, C. S. 1931. Collected Papers, ed. by C. Hartshorne and P. Weiss, vol.2. Cambridge, Mass.: Harvard University Press.

Petitot, E. F. S. 1876. Dictionnaire de la langue Dènè-Dindjié, dialectes Montagnais ou Chippe way an, peaux de Lièvre et Loucheux, Précédé d'une monographie des Dènè-Dindjié, d'une grammaire et de tableaux synoptiques des conjugaisons. Paris, Bibliothèque de linguistique et d'ethnographie Américaine. Vol.II.

Saxe, G. B. 1981. "Body parts as numerals: a developmental analysis of numeration among the Oksapmin in Papua New Guinea”. Child Development 52: 306-316.

Spelke, E. S. 1990. "Principles of object perception”. Cognitive Science 14: 29-56.

Strehlow, T. G. H. ca. 1945. Aranda Phonetics and Grammar [The Oceania monographs 7]. Sidney: Australian National Research Council.

Suzuki, K., and Kobayashi, T. 2000. "Numerical competence in rats (Rattus norvegicus): Davis and Bradford (1986) extended”. Journal of Comparative Psychology 114 (1): 73-85. 
Uller, C.; Carey, S.; Huntley-Fenner, G., and Klatt, L. 1999. "What representations might underlie infant numerical knowledge?” Cognitive Development 14 (1): 1-36.

Whalen, J.; Gallistel, C. R., and Gelman, R. 1999. "Nonverbal counting in humans: The psychophysics of number representation”. Psychological Science 10 (2): 130-137.

Wiese, H. 2003a. Numbers, Language, and the Human Mind. Cambridge: Cambridge University Press.

Wiese, H. 2003b. "Iconic and non-iconic stages in number development: the role of language”. Trends in Cognitive Sciences 7 (9): 385-390.

Wynn, K. 1990. “Children's understanding of counting. Cognition 36 (2): 155-193.

Wynn, K. 1992a. "Addition and subtraction by human infants". Nature 358: 749-750.

Wynn, K. 1992b. “Children's acquisition of the number words and the counting system.” Cognitive Psychology 24: 220-251.

Wynn, K. 1998. "Numerical competence in infants". In The Development of Mathematical Skills, C. Donlan (ed.), 3-25. Hove: Psychology Press.

Wynn, K.; Bloom, P., and Chiang, W.-C. 2002. "Enumeration of collective entities by 5-month-old infants”. Cognition 83: B55B62. 